\title{
3-D SEISMIC MODELING AND DEPTH MIGRATION COMBINING THE EXTRAPOLATION OF UPGOING AND DOWNGOING WAVEFIELDS
}

\author{
Gary Corey Aldunate ${ }^{1}$ and Reynam C. Pestana ${ }^{2}$
}

\begin{abstract}
The 3-D acoustic wave equation is generally solved using finite difference schemes on the mesh which defines the velocity model. However, when numerical solution of the wave equation is done by finite difference schemes, attention should be taken with respect to dispersion and numerical stability. To overcome these problems, one alternative is to solve the wave equation in the Fourier domain. This approach is stabler and makes possible to separate the full wave equation in its unidirectional equations. Thus, the full wave equation is decoupled in two first order differential equations, namely two equations related to the vertical component: upgoing (-Z) and downgoing (+Z) unidirectional equations. Among the solution methods, we can highlight the Split-Step-Plus-Interpolation (SS-PSPI). This method has been proven to be quite adequate for migration problems in 3-D media, providing satisfactory results at low computational cost. In this work, 3-D seismic modeling is implemented using Huygens' principle and an equivalent simulation of the full wave equation solution is obtained by properly applying the solutions of the two uncoupled equations. In this procedure, a point source wavefield located at the surface is extrapolated downward recursively until the last depth level in the velocity field is reached. A second extrapolation is done in order to extrapolate the wavefield upwards, from the last depth level to the surface level, and at each depth level the previously stored wavefield (saved during the downgoing step) is convolved with a reflectivity model in order to simulate secondary sources. To perform depth pre-stack migration of 3-D datasets, the decoupled wave equations were used and the same process described for seismic modeling is applied for the propagation of sources and receivers wavefields. Thus, depth migrated images are obtained using appropriate image conditions: the upgoing and downgoing wavefields of sources and receivers are correlated and the migrated images are formed. The seismic modeling and migration methods using upgoing and downgoing wavefields were tested on simple 3-D models. Tests showed that the addition of upgoing wavefield in seismic migration, provide better result and highlight steep deep reflectors which do not appear in the results using only downgoing wavefields.
\end{abstract}

Keywords: 3-D seismic modeling and migration, Upoing and downgoing wavefields, Split-Step Phase Shift Plus Interpolation method, Decoupled wave equations, One-Way equations.

RESUMO. A equação da onda acústica tridimensional é normalmente resolvida usando-se esquemas de diferenças finitas sobre a malha que define 0 modelo de velocidade. Entretanto, deve-se ter cuidado com a dispersão e a estabilidade numérica durante o processo de propagação da onda na malha. Uma outra alternativa, bastante eficiente de se resolver a equação completa da onda, é desacoplando-a em duas equações de onda unidirecionais no domínio transformado de Fourier (solução pseudo-espectral). Assim, a equação completa da onda é separada em duas equações diferenciais de primeira ordem relativa à componente vertical: equação da onda ascendente (-Z) e da onda descendente (+Z). Normalmente, a equação unidirecional é resolvida com diferentes ordens de aproximação. Entre esses métodos, podemos destacar o método "Split-Step-Plus-Interpolation" (SS-PSPI), que tem sido bastante adequado para problemas de migração em meios 3-D, fornecendo resultados a um baixo custo computacional. Neste trabalho, o modelamento sísmico 3-D foi implementado usando-se o princípio de Huygens com as duas equações de onda unidirecionais desacopladas. Com o objetivo de simular uma solução equivalente à solução da equação completa, uma fonte pontual localizada na superfície é extrapolada em profundidade, de forma recursiva, até atingir o último nível de profundidade na malha do modelo de velocidades. Uma segunda extrapolação é realizada para extrapolar para cima o campo de onda, desde o último nível em profundidade até à superfície do modelo. Assim, os receptores localizados na superfície registram 0 campo de onda ascendente, que trazem informações dos refletores em subsuperfície na forma de reflexões e difrações. Para realizar a migração pré-empilhamento em profundidade de dados 3-D, usando-se as equações de onda desacopladas, o mesmo procedimento descrito para o modelamento sísmico é utilizado para a propagação dos campos de onda de fontes e receptores. Imagens migradas são obtidas usando-se condições de imagem apropriadas, onde os campos de onda da fonte e dos receptores, descendente e ascendente, são correlacionados. Sobre modelos 3-D simples foram testados os métodos de modelamento e migração, levando em conta os campos de onda ascendente e descendente. Ficando, assim, evidenciado que no método de migração sísmica, proposto aqui, a adição do campo de onda ascendente fornece um melhor resultado, ressaltando os refletores íngremes que não aparecem nos resultados utilizando-se apenas a extrapolação do campo de onda descendente.

\footnotetext{
Palavras-chave: Migração e modelagem sísmica 3-D, Migração em duas etapas mais interpolação, equações de ondas unidirecionais.

${ }^{1}$ Universidade Federal da Bahia, Centro de Pesquisa em Geofísica e Geologia (CPGG), Rua Barão de Jeremoabo, 123, Campus Universitário da Federação, 40170-115 Salvador, BA, Brazil. Phone: +55(71) 9244-1768 - E-mail: aldunategary@gmail.com

2 Universidade Federal da Bahia, Departamento de Geofísica da Terra e do Meio Ambiente, Instituto de Física/CPGG, Rua Barão de Jeremoabo, 123, Campus Universitário da Federação, 40170-115 Salvador, BA, Brazil. Phone: +55(71) 3283-8521; Fax: +55(71) 3283-8551 - E-mail: reynam@cpgg.ufba.br
} 


\section{INTRODUCTION}

Seismic modeling plays an important role when processing and interpreting seismic data. Modeling is the basis for the pre-stack seismic migration which aims to obtain a subsurface image from the dataset recorded at the acquisition surface. The seismic modeling methods belong to two main categories: the first is based on the integral solution of the wave equation and is commonly called ray tracing. These methods are computationally efficient, but present low accuracy on structurally complex models. The second, which are based on the full wave equation and explicit finite difference schemes, on the other hand, can generate more accurate data, make possible to model seismic events such as returning waves, duplex waves and multiple arrivals events. For these methods the full acoustic wave equation is solved with time as the preferred domain of evolution of the seismic waves. However, special attention should be taken into account while the wavefield is propagated in time due to dispersion and stability problems, which are typical from explicit finite difference methods (Thorbecke, 2012).

Recently, Xiaojun et al. (2006) and Wu et al. (2007) proposed a modeling scheme using the one-way wave equation for common shot gathers, where extrapolation of the wavefield can be made to any arbitrary depth in the model. Here, in this work, for the modeling and migration of the seismic data, we present the decoupling of the 3-D full wave equation into two unidirectional wave equations (Amundsen et al., 2008; Zhang \& Wong, 2010). To place the modeling, we use the solution of the two unidirectional equations (Zhang et al., 2006) and apply Huygens' principle, adding a secondary source to one of the unidirectional equations, as suggested by Wu et al. (2007). The one-way extrapolation operator used is the Split-Step-Plus-Interpolation - SS-PSPI (Chen \& Du, 2010; Aldunate \& Pestana, 2010, 2011). This operator was chosen because it is robust, can deal very well with lateral velocity variations, and has no azimuthal anisotropy in 3-D media. The algorithm implemented for the modeling is quite simple, computationally efficient and produces no interference of multiple reflections or direct wave. Moreover, in simple models, the targets can be readily recognizable as events in the seismogram recorded at the surface of the model.

In the 3-D modeling process of common shot sections, a point source is injected into the surface of the model and the extrapolation of this wavefield associated with this source is performed recursively for all depth levels in the velocity model. During the downward extrapolation process in depth, the propagated wavefield is stored for every depth level. That is, for each $(X-Y)$ plane. The downward extrapolation is taken as the positive direc- tion, and called downward propagation or just "Down". To simulate the wavefield in the upward direction, from the last depth level until the surface of the model, the wavefield previously stored at each depth level of the model is convolved with a reflectivity function and upward propagated. The wavefield at each depth level now acts as a new secondary source, whose intensity is dominated by the amplitude of the reflectivity at each grid point. The reflectivity function is calculated from the velocity model. At the end of the modeling process, the receivers along the surface record the information from the upward wavefield. Thus, the seismic modeling is carried out using two undirectional wave equations.

It is also worth noting the following advantages of this modeling based on the principle of Huygens and the 3-D unidirectional SS-PSPI operators, compared with finite difference methods: (1) the source can be located exactly on the surface; (2) the signature of the source can be zero phase without dispersion effect in the modeled section; (3) the modeled section is not contaminated with the direct wave; (4) multiple scattering events are eliminated as the type peg-legs, which are very well simulated with the solution of the full acoustic wave equation by finite difference schemes; (5) due to the fact that the spatial derivatives in the solution of the unidirectional wave equation are calculated in the spectral domain, stability problems are not observed in the evolution of the waves in the mixed domain frequency-wavenumber space; (6) the modeling can generate both duplex and returning waves.

These same uncoupled unidirectional equations can be used for migrating 3-D seismic data and with a higher accuracy than the conventional migration, producing the imaging of reflectors with angles greater than $90^{\circ}$ and associated to high lateral velocity variations. The process for migration is similar to the modeling, Huygens' principle is again applied, that is, source and receivers wavefield are stored up at each depth level in the down extrapolation procedure. Then these stored wavefield are extrapolated upward recursively, during the second passage. Finally, for the image formation in depth, appropriate imaging conditions are applied to correlate the wavefields of the source (up and down) and receivers (up and down) (Jin \& Xu, 2006; Ye \& Jia, 2012).

To illustrate and validate the seismic modeling method proposed in this work some 3-D common shot gathers were modeled using two different 3-D velocity models and recorded along the surface the model. Then, these two datasets were used to perform pre-stack depth migration. Afterwards, the numerical test results were analyzed to identify the events recorded in the modeling process, as well as to verify the correct spatial position of the reflectors imaged by the pre-stack migration method proposed in this paper. The 3-D migration procedure, that takes into account the 
propagation steps of Up-Down waves using the SS-PPSI method will be from now and on called SS-PSPI-UP-DOWN method. We also demonstrate that this method makes possible to image reflectors with slope beyond ninety degrees and the results were superior to those obtained by the SS-PSPI conventional method that considers during the entire extrapolation process only the downward wavefields.

\section{UNCOUPLING OF THE 3-D ACOUSTIC WAVE EQUATION}

The full acoustic wave equation for 3-D media is written as:

$$
\frac{\partial^{2} p}{\partial z^{2}}+\frac{\partial^{2} p}{\partial x^{2}}+\frac{\partial^{2} p}{\partial y^{2}}=\frac{1}{v^{2}(y, x, z)} \frac{\partial^{2} p}{\partial t^{2}},
$$

where $x, y, z$ are the spatial variables, $t$ is the time, $p(t, y, x, z)$ is the acoustic pressure wavefield and $v(y, x, z)$ is the propagation velocity in the medium.

To start the decoupling process of equation (1) in two first order partial differential equations, we first apply a Fourier transform in time, thereby obtaining the full wave equation in the frequencyspace domain:

$$
\frac{\partial^{2} P}{\partial z^{2}}+\frac{\partial^{2} P}{\partial x^{2}}+\frac{\partial^{2} P}{\partial y^{2}}=-\frac{\omega^{2}}{v^{2}(y, x, z)} P,
$$

where $P=P(\omega, y, x, z)$ and $\omega$ is the frequency. The pressure field $P(\omega, y, x, z=0)$ is known, but, $\frac{\partial P}{\partial z}$ in $z=0$ is unknown, so, it is difficult to solve the equation (2), since that the two boundary conditions are required. To overcome this difficulty, we rewrite equation (2) putting in evidence the $Z$ direction as the preferred direction of propagation, obtaining then:

$$
\frac{\partial^{2} P}{\partial z^{2}}=-\left(\frac{\omega^{2}}{v^{2}(y, x, z)}+\frac{\partial^{2}}{\partial x^{2}}+\frac{\partial^{2}}{\partial y^{2}}\right) P .
$$

Rewriting equation (3) in matrix notation, we have:

$$
\frac{\partial}{\partial z}\left[\begin{array}{c}
P \\
\frac{\partial P}{\partial z}
\end{array}\right]=\left[\begin{array}{cc}
0 & 1 \\
-S^{2} & 0
\end{array}\right]\left[\begin{array}{c}
P \\
\frac{\partial P}{\partial z}
\end{array}\right],
$$

where

$$
S^{2}=\left(\frac{\omega^{2}}{v^{2}(y, x, z)}+\frac{\partial^{2}}{\partial x^{2}}+\frac{\partial^{2}}{\partial y^{2}}\right)
$$

Naming the first array on the right hand side of equation (4), as:

$$
A=\left[\begin{array}{cc}
0 & 1 \\
-S^{2} & 0
\end{array}\right]
$$

where the matrix $A$ can be diagonalized using its eigenvectors and eigenvalues, as:

$$
A=V \Lambda V^{-1}
$$

where $V, \Lambda, V^{-1}$ are defined, respectively, as:

$$
\begin{gathered}
V=\left[\begin{array}{cc}
1 & 1 \\
-i S & i S
\end{array}\right], \\
\Lambda=\left[\begin{array}{cc}
-i S & 0 \\
0 & i S
\end{array}\right], \\
V^{-1}=\frac{1}{2}\left[\begin{array}{cc}
1 & i S^{-1} \\
1 & -i S^{-1}
\end{array}\right],
\end{gathered}
$$

where $V$ is composed of the eigenvectors associated to the matrix $A, \Lambda$ the eigenvalues of $A$ and $V^{-1}$ is the inverse of $V$.

Applying the Fourier transform to the spatial variables $y, x$ in the wave equation (3), and considering that $v=v_{0}(z)$, which does not allow lateral variations, we obtain the following equation in frequency-wavenumber domain:

$$
\frac{\partial^{2} P}{\partial z^{2}}=-\left(\frac{\omega^{2}}{v_{0}^{2}(z)}-k_{x}^{2}-k_{y}^{2}\right) P,
$$

where $P=P\left(\omega, k_{y}, k_{x}, z\right)$ and $k_{x}, k_{y}$ are the horizontal wavenumbers. The dispersion relation, i.e, the vertical wavenumber $k_{z}$, is defined as:

$$
k_{z}^{2}=\frac{\omega^{2}}{v_{0}^{2}(z)}-k_{x}^{2}-k_{y}^{2} .
$$

Then, equation (10) can be expressed as:

$$
\left(\frac{\partial^{2}}{\partial z^{2}}+k_{z}^{2}\right) P=\left(\frac{\partial}{\partial z}+i k_{z}\right)\left(\frac{\partial}{\partial z}-i k_{z}\right) P=0 .
$$

Writing equation (12) in frequency-space domain, we have:

$$
\left(\frac{\partial}{\partial z}+i K_{z}\right)\left(\frac{\partial}{\partial z}-i K_{z}\right) P=0
$$

where the new dispersion relation $\left(K_{z}\right)$ in this domain, is given by the expression:

$$
K_{z}=\sqrt{\frac{\omega^{2}}{v^{2}(y, x, z)}+\frac{\partial^{2}}{\partial x^{2}}+\frac{\partial^{2}}{\partial x^{2}}} .
$$

Now, decomposing the total wavefield $P$ into a down wavefield $D$ and a up wavefield $U$, as:

$$
P=D+U
$$


and considering the equations for $D$ and $U$ from equation (13)

$$
\begin{aligned}
& \left(\frac{\partial}{\partial z}+i K_{z}\right) D=0, \\
& \left(\frac{\partial}{\partial z}-i K_{z}\right) U=0,
\end{aligned}
$$

we can add equations (16) and (17) taking into account equation (15) to obtain:

$$
\begin{gathered}
\frac{\partial}{\partial z} D+\frac{\partial}{\partial z} U=-i K_{z} D+i K_{z} U \\
\frac{\partial}{\partial z}(D+U)=-i K_{z} D+i K_{z} U
\end{gathered}
$$

and

$$
\frac{\partial P}{\partial z}=-i K_{z}(D-U)
$$

Expressing equations (15) and (19) in matrix notation and taking advantage of the eigenvalues of $A$, we obtain:

$$
\left[\begin{array}{c}
P \\
\frac{\partial P}{\partial z}
\end{array}\right]=V\left[\begin{array}{c}
D \\
U
\end{array}\right] .
$$

Therefore, the complete acoustic wave equation written in matrix notation, equation (4), can be rewritten into its components $D$ and $U$, as:

$$
\begin{aligned}
\frac{\partial}{\partial z} V\left[\begin{array}{l}
D \\
U
\end{array}\right] & =\left(V \Lambda V^{-1}\right) V\left[\begin{array}{l}
D \\
U
\end{array}\right], \quad \text { or, } \\
\frac{\partial}{\partial z}\left[\begin{array}{l}
D \\
U
\end{array}\right] & =\left(\Lambda-V^{-1} \frac{\partial V}{\partial z}\right)\left[\begin{array}{l}
D \\
U
\end{array}\right] .
\end{aligned}
$$

Thus we obtain the following decoupled equations for the components $D$ and $U$ :

$$
\begin{aligned}
& \frac{\partial D}{\partial z}=-i K_{z} D-\frac{1}{2 K_{z}} \frac{\partial K_{z}}{\partial z}(D-U) \\
& \frac{\partial U}{\partial z}=+i K_{z} U+\frac{1}{2 K_{z}} \frac{\partial K_{z}}{\partial z}(D-U) .
\end{aligned}
$$

Equations (23) and (24) represent unidirectional wave equations, which can be used for wavefield extrapolation in inhomogeneous media. The extrapolation can be implemented using the above equations, however, the downward and upward wavefield are still coupled. During the extrapolation process, we note that the down wavefield depends on the up wavefield while the up wavefield is also required when the descending wavefield is extrapolated. Then the extrapolation process is not easy to be implemented due to this type of coupling between the wavefields. In the case where the wavefield $D$ has just incident wave at an angle of incidence lower than the critical angle, the reflected wavefield amplitude is small compared to the amplitude of $U$. In the same way, the amplitude of $U$ is small compared with the amplitude of the wavefield $D$. Thus, for angles of incidence below the critical angle, the fields $U$ and $D$ do not consider the multiple scattering of seismic energy.

Disregarding the coupling of the upward and downward wavefields, the relations (23) and (24) can be simplified to

$$
\begin{aligned}
& \frac{\partial D}{\partial z}=-i K_{z} D-\frac{1}{2 K_{z}} \frac{\partial K_{z}}{\partial z}(D) \\
& \frac{\partial U}{\partial z}=+i K_{z} U+\frac{1}{2 K_{z}} \frac{\partial K_{z}}{\partial z}(-U) .
\end{aligned}
$$

The equations (25) and (26) represent the unidirectional uncoupled equations obtained from the complete wave equation, and are equations for the descending and ascending wavefields, respectively. If the medium is homogeneous along $Z$ direction, then $\frac{\partial K_{z}}{\partial z}=0$. So, equations (25) and (26) can be further reduced to:

$$
\begin{aligned}
& \frac{\partial D}{\partial z}=-i K_{z} D \\
& \frac{\partial U}{\partial z}=+i K_{z} U .
\end{aligned}
$$

Thus, using only a boundary condition, one can solve the unidirectional wave equations (27) and (28), which can also be valid for non-homogeneous media, because $K_{z}$ contains the velocity $v(x, y, z)$ without any restrictions.

After applying the Fourier transform to equations (27) and (28), and already in the frequency-wavenumber domain $\left(\omega, k_{x}, k_{y}\right)$, the full wave equation separated in its unidirectional equations (directions $-Z$ and $+Z$ ), considers a medium without lateral velocity changes, i.e, $v=v_{0}(z)$. The medium is for each depth level assumed homogeneous in the $X$ and $Y$ directions and therefore just one velocity is needed. These equations are expressed in terms of the vertical wavenumber $k_{z}$ as:

$$
\begin{aligned}
& \frac{\partial D}{\partial z}=-i k_{z} D \\
& \frac{\partial U}{\partial z}=+i k_{z} U
\end{aligned}
$$

Equations (29) and (30) are partial differential equations of first 
order and have, respectively, the following solutions:

$$
\begin{aligned}
D(z) & =e^{-i k_{z} z} D(0) \\
& =e^{-i \frac{\omega}{v_{0}(z)} \sqrt{1-\frac{v_{0}^{2}(z)}{\omega^{2}}\left(k_{x}^{2}+k_{y}^{2}\right)} z} D(0) . \\
U(z) & =e^{+i k_{z} z} U(0) \\
& =e^{+i \frac{\omega}{v_{0}(z)} \sqrt{1-\frac{v_{0}^{2}(z)}{\omega^{2}}\left(k_{x}^{2}+k_{y}^{2}\right)}} z(0) .
\end{aligned}
$$

\section{3-D SPLIT-STEP-PLUS-INTERPOLATION (SS-PSPI) OPERATOR}

One way to overcome the restrictions of lateral homogeneity of the solutions expressed in equations (31) and (32) is to take in consideration the velocity variations in the lateral directions $Y$ and $X$, i.e. $v=v(y, x, z)$. Thus, there will be a domain conflict, since in the square root term the velocity is in the spatial domain and the spatial derivatives are calculated in the transformed Fourier domain, that is, as function of the spatial wavenumbers. To overcome this conflict, we propose to use the SS-PSPI method (Aldunate \& Pestana, 2006, 2010, 2011; Chen \& Du, 2010), where the extrapolation operator for each temporal frequency is written as independent functions. Applying the SS-PSPI method for the operators $D$ and $U$, equations (31) and (32), we obtain:

$$
\begin{aligned}
& \mathcal{D}\left(x, y ; k_{x}, k_{y}\right) \sim \sum_{j=1}^{n} f_{j}^{D}(x, y) g_{j}^{D}\left(k_{x}, k_{y}\right), \\
& \mathcal{U}\left(x, y ; k_{x}, k_{y}\right) \sim \sum_{j=1}^{n} f_{j}^{U}(x, y) g_{j}^{U}\left(k_{x}, k_{y}\right),
\end{aligned}
$$

where $\mathcal{D}$ and $\mathcal{U}$ represent the 3-D SS-PSPI operator downward and upward, respectively. The index $j$, with $j=1, \ldots, n$, indicates the $n$ reference velocities used in the solutions (31) and (32), thus, the operator is applied $n$ times, one for each reference velocity: $v_{0_{1}}, v_{0_{2}}, \ldots, v_{0_{n}}$. Therefore, it improves the accuracy of the operator in horizontal directions $X$ and $Y$, because only a reference velocity would not be enough to handle strong lateral velocity variations. The function $g_{j}\left(k_{x}, k_{y}\right)$ is responsible for the phase-shift in the transformed Fourier domain $\left(w, k_{y}, k_{x}\right)$, and has the following form:

$$
g_{j}\left(k_{x}, k_{y}\right)=e^{ \pm i \frac{\omega}{v_{0_{j}}(z)} \sqrt{1-\frac{v_{0_{j}}^{2}(z)}{\omega^{2}}\left(k_{x}^{2}+k_{y}^{2}\right)} z},
$$

where the negative sign corresponds to the operator $\mathcal{D}$ and the positive to $\mathcal{U}$. After an inverse spatial Fourier transform, i.e., in the frequency-space domain $(\omega, y, x)$ a time shift is applied (split-step correction) to take into account the lateral velocity variations. The time shift with the interpolation is given by:

$$
f_{j}(x, y)=h_{j}(x, y) e^{ \pm i \omega\left(\frac{1}{v(y, x, z)}-\frac{1}{v_{0_{j}}}\right) z},
$$

where $h_{j}(x, y)$ represents the interpolation function of the $n$ wavefields. The technique for selecting reference velocities used in this work was presented by Aldunate \& Pestana (2010).

Thus, the 3-D SS-PSPI operator is applied for each fixed frequency, through the following steps: (1) phase-shift correction in the wavenumber domain given by $g_{j}\left(k_{x}, k_{y}\right)$; (2) spatial Fourier transform, followed by a temporal phase correction in the frequency-space domain using $f_{j}(x, y)$.

\section{SEISMIC MODELING USING THE 3-D SS-PSPI-UP-DOWN OPERATOR}

Seismic modeling is a technique for simulating the propagation of acoustic wave inside the Earth. The goal is to simulate the response that would be recorded by seismic receivers located on the surface, given a defined subsurface structure. This technique is widely used in seismic interpretation and is an essential part of the seismic inversion algorithms. Another important application of seismic modeling is evaluation and planning of seismic acquisitions (Carcione et al., 2002). Through modeling and pre-stack depth migration the dataset and its seismic acquisition parameters can be evaluated before the acquisition process.

In seismic modeling process, the kinematic point of view, the seismic wave propagates in accordance with the Huygens' principle, and in any instant of time the wavefront can be seen as a new set of radiating sources. In turn, the geological structure of the subsurface is represented by a model of consistent velocities with a assigned velocity mesh at each point. Velocities whose values are different between neighbour mesh points define an interface or a reflector. Thus, the presence of an interface indicates change of acoustic impedance. The reflectivity at each point of the velocity model is computed from its spatial derivative as:

$$
r(y, x, z)=\sqrt{\left(\frac{\partial v}{\partial y}\right)^{2}+\left(\frac{\partial v}{\partial x}\right)^{2}+\left(\frac{\partial v}{\partial z}\right)^{2}} .
$$

Thus, a reflective surface of greater amplitude is represented by a higher impedance (strong velocity contrast) and, therefore, these model points reflect more energy than the other mesh points where the velocity is constant or presents a weakly velocity variations, thus, it is a region in which the reflectivity has a small amplitude. 
With these considerations, the acoustic modeling in 3-D medium using unidirectional operators given by equations (33) and (34), is made through a recursive extrapolation of the wavefield (that contains a source term: $F$ ) from the surface level until it reaches the deepest level over the mesh that defines the velocity model. Then, that same wavefield is propagated upwards (acting as a secondary source), recursively until it reaches the surface of the model. The upward propagation part takes into account the reflectivity which is convolved with the wavefield at each point of the model, and thus, it has been incorporated into the propagation of the ascending and descending wavefields using unidirectional operators. This whole modeling process is described by the following equations:

$$
\left\{\begin{array}{l}
\frac{\partial D}{\partial z}+i k_{z} D=-F \\
\frac{\partial U}{\partial z}-i k_{z} U=D \otimes r
\end{array}\right.
$$

where $\otimes$ denotes the convolution operation.

The seismic modeling process is achieved by the following equation

$$
\begin{gathered}
S_{D}\left(\omega, x, y, z_{0}+\Delta z\right)= \\
=\sum_{j=1}^{n} h_{j}(x, y) \cdot f_{j}(x, y) \\
\times\left[\mathcal{F}_{x, y}^{-1}\left\{g_{j}\left(k_{x}, k_{y}\right) \cdot\left[\mathcal{F}_{x, y}\left\{S\left(\omega, y, x, z_{0}\right)\right\}\right]\right\},\right.
\end{gathered}
$$

where $\mathcal{F}_{x, y}$ and $\mathcal{F}_{x, y}^{-1}$ represent the direct and inverse Fourier transform, respectively.

The process starts with the source wavefield, $S(\omega, y, x, z=$ 0 ), that contains a Ricker zero phase-type wavelet and is defined by function $(F)$, at the position $\left(x_{s}, y_{s}, z=0\right)$. Initially, it is transformed into the domain $\left(w, k_{y}, k_{x}\right)$ through a 2-D forward Fourier transform $-\left(\mathcal{F}_{x, y}\right)$. Then the source wavefield is multiplied by $g_{j}\left(k_{x}, k_{y}\right)$, which corresponds to the phase-shift correction. After that, it is back transformed to the domain $(w, y, x)$ through a 2-D inverse Fourier transform $-\left(\mathcal{F}_{x, y}^{-1}\right)$. With the source wavefield in this domain, the lateral velocity is corrected by $f_{j}(x, y)$, which is called the split-step correction. Finally, an interpolation procedure is applied using all wavefields through the interpolation function $h_{j}(x, y)$. Thus, we obtain the source wavefield in the next level $\left(z_{0}+\Delta z\right)$. The whole process described above can be reduced by the following compact notation, where it is represented by the application of the operator $\mathcal{D}$ to the source wavefield as:

$$
S_{D}\left(\omega, x, y, z_{0}+\Delta z\right)=\mathcal{D}\left\{S\left(\omega, y, x, z_{0}\right)\right\} .
$$

Figure 1(a) illustrates the source wavefield downgoing extrapolation process starting at the surface.
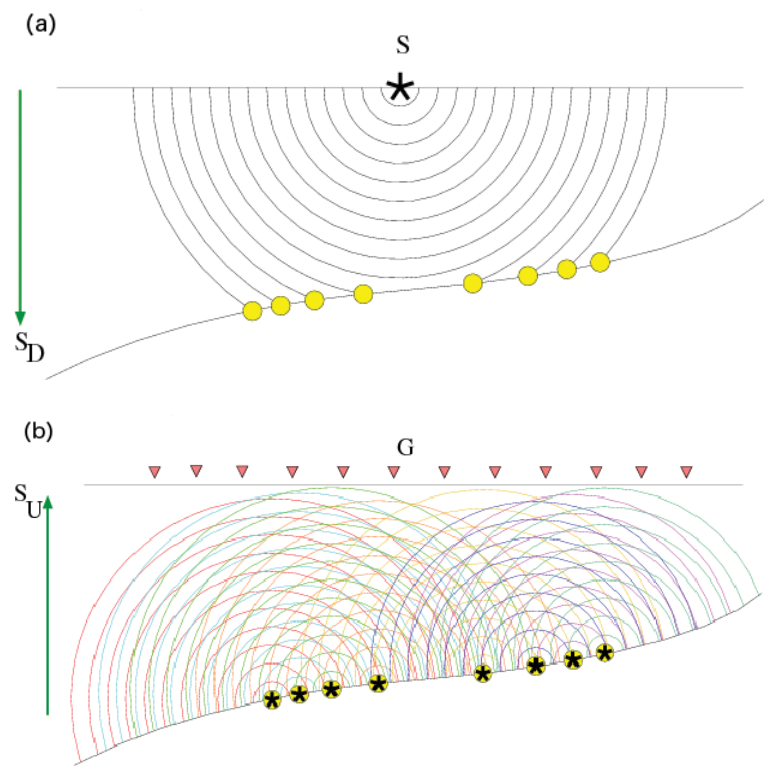

Figure 1 - Huygens' principle - (a) A point source is injected at the surface of the model and each point in the model constitutes a new source for propagation of waves down. (b) During the upward extrapolation, using the same principle, each point along the reflector is considered as a secondary source for the propagation of waves upwards.

The downward propagation of the source wavefield is carried out recursively until it reaches the maximum, $N-1$ depth levels, indicated by: $z_{\max }=z_{N-2}+\Delta z$. During this process of downward continuation at all depths, the source wavefield $S_{D}\left(\omega, x, y, z_{k}\right)$ is saved, with $k=0,1, \ldots, N-1$.

Once in the level $z_{\max }=z_{N-1}$, the source wavefield is propagated again, now upward, using the operator $\mathcal{U}$, which is given by equation (34). According to Huygens' principle, at any instant of time the wavefront propagates also in upward direction and thus generating secondary sources in all points of the model. To take this in account, during the upward propagation the reflectivity function is convolved with the extrapolated upward wavefield and added to the field $S_{D}\left(\omega, x, y, z_{k}\right)$. Thus, the resulting wavefield at each depth level also includes the wavefield that was downward propagated. To perform the upward extrapolation starting from the last level in depth, it considers that: $S_{U}\left(\omega, x, y, z_{N-1}\right)=S_{D}\left(\omega, x, y, z_{N-1}\right)$. Therefore, the upward propagation is done through:

$$
\begin{gathered}
S_{U}\left(\omega, x, y, z_{N-1}-\Delta z\right)= \\
=\mathcal{U}\left\{S_{U}\left(\omega, y, x, z_{N-1}\right) r\left(y, x, z_{N-1}\right)\right. \\
\left.+S_{D}\left(\omega, y, x, z_{N-1}\right)\right\}
\end{gathered}
$$


until the surface $z_{0}=z_{1}-\Delta z$ of the model is reached. The upward propagation procedure using Huygens' principle is shown in Figure 1(b).

On the surface of the model, the ascending wavefield is registered in the matrix $G(w, y, x, z=0)$, wherein at each point of the surface $\left(x_{r}, y_{r}, z=0\right)$ there is a receptor.

The propagation scheme is repeated for each frequency $\omega$. All frequencies are summed and accumulated in the same matrix $G$. Then, an inverse temporal Fourier transform is applied to $G$, generating a recorded section in the time domain. That is,

$$
\begin{gathered}
G\left(t, x_{r}, y_{r}, z=0\right)= \\
=\mathcal{F}_{t}^{-1}\left(\sum_{\omega} G\left(\omega, x_{r}, y_{x}, z=0\right)\right) .
\end{gathered}
$$

In the plane $z=0$ and on each surface point $\left(x_{r}, y_{r}, z=0\right)$ we will have a seismic trace recorded.

Seismograms and 3-D snapshots, obtained with this modeling procedure, are shown for different models and an analysis and a detailed discussion of these numerical results are presented in the numerical results section.

\section{3-D SS-PSPI-UP-DOWN PRE-STACK DEPTH MIGRATION}

The 3-D migration with conventional unidirectional operators is performed by a downgoing extrapolation along the $+Z$ directions of sources and receivers followed by the cross-correlation imaging condition of sources and receivers wavefields at each depth level. To perform seismic migration with the full wavefield propagation (without taking into account the multi-scattering) the same seismic modeling procedure described previously is applied. For migration, in addition to propagate the source wavefield, the recorded data along the receivers is also propagated. So, the migration procedure consists in save source wavefield $\left(S_{D}\right)$ and receivers wavefield $\left(G_{D}\right)$, at each depth level $z_{k}$, (during the downward extrapolation). Then, those stored wavefield in each $z_{k}$ level act as a new secondary source and both are upward extrapolated using the operator $\mathcal{U}$. At each $z_{k}$ depth level the imaging conditions is applied. It aims to correlate the sources and receivers wavefields, which will form migrated images in depth. The system of equations that describe this procedure is:

$$
\left\{\begin{array}{l}
\frac{\partial D}{\partial z}+i k_{z} D=-F \\
\frac{\partial U}{\partial z}-i k_{z} U=D .
\end{array}\right.
$$

The migration process begins when the data recorded in the time domain, $G\left(t, x_{r}, y_{r}, z=0\right)$, is transformed to the frequency domain. With the implementation of the down extrapolation operator, equation (33), one obtains the data wavefield at a depth level below, thus, it is extrapolated for a level $z+\Delta z$, expressed as:

$$
G_{D}(\omega, x, y, z+\Delta z)=\mathcal{D}\{G(\omega, x, y, z)\} .
$$

Once covered all depth levels and considering that in the last depth level $G_{U}\left(\omega, x, y, z_{N-1}\right)=G_{D}\left(\omega, x, y, z_{N-1}\right)$, the receiver wavefield is extrapolated upward, applying the ascending operator $\mathcal{U}$, by the following equation:

$$
\begin{gathered}
G_{U}\left(\omega, y, x, z_{N-1}-\Delta z\right)= \\
=\mathcal{U}\left\{G_{U}\left(\omega, y, x, z_{N-1}\right)+G_{D}\left(\omega, y, x, z_{N-1}\right)\right\},
\end{gathered}
$$

where in this part of the extrapolation process the receiver wavefield is not convolved with the reflectivity function. In our method, the reflectivity is estimated based on the knowledge of the velocity field. However, in most of the cases, the velocity model used for migration is an approximated version of it. Thus, there is no absolute certainty of the reflector location in the velocity model used for migration.

Likewise, the wavefield of the source, $S(\omega, x, y, z=0)$, associated with a Ricker pulse is injected over the acquisition surface of the model and then propagated on the velocity mesh. The downward extrapolation of the source wavefield is carried out by:

$$
S_{D}(\omega, x, y, z+\Delta z)=\mathcal{D}\{S(\omega, x,, z)\} .
$$

Again, starting form the last depth level $\left(z_{N-1}\right)$, the same procedure is applied and considering that in the last depth level $S_{U}=S_{D}$, the upward propagation is given by:

$$
\begin{gathered}
S_{U}\left(\omega, x, y, z_{N-1}-\Delta z\right)= \\
=\mathcal{U}\left\{S_{U}\left(\omega, x, y, z_{N-1}\right)+S_{D}\left(\omega, x, y, z_{N-1}\right)\right\} .
\end{gathered}
$$

The whole process at the end of the migration procedure, as described above, results in four wavefields: $G_{D}, G_{U}, S_{D}$ and $S_{U}$.

Now, an imaging condition needs to be applied to obtain the migrated section. The imaging condition for common shot data is usually applied taken the cross-correlation of the source $(S)$ and receivers wavefield $(G)$ for all depth level and it is expressed as (Claerbout, 1985):

$$
I(y, x, z)=\sum_{\omega} S^{*}(\omega, y, x, z) \cdot G(\omega, y, x, z),
$$

where $(y, x, z)$ is the spatial location of an image point in the 3 -D volume and $*$ represents the complex conjugate.

As the $S$ and $G$ wavefields were decomposed in its descending and ascending wavefields, the correlation imaging condition, 
equation (47), can be now rewritten as:

$$
\begin{gathered}
I(y, x, z)= \\
=\sum_{\omega}\left[S_{D}(\omega, y, x, z)+S_{U}(\omega, y, x, z)\right]^{*} \\
\times\left[G_{D}(\omega, y, x, z)+G_{U}(\omega, y, x, z)\right],
\end{gathered}
$$

and the possible imaging conditions for all these wavefields are:

$$
I_{D D}(y, x, z)=\sum_{\omega} S_{D}^{*}(\omega, y, x, z) \cdot G_{D}(\omega, y, x, z),
$$

where this is the conventional imaging condition, using only the downward propagated wavefields.

The image obtained by:

$$
I_{D U}(y, x, z)=\sum_{\omega} S_{D}^{*}(\omega, y, x, z) \cdot G_{U}(\omega, y, x, z)
$$

will image reflectors with dips greater than ninety degrees, since it takes into account the receptor wavefield propagated upwards correlated with the downward wavefield of the source.

In a similar way, the imaging condition

$$
I_{U D}(y, x, z)=\sum_{\omega} S_{U}^{*}(\omega, y, x, z) \cdot G_{D}(\omega, y, x, z)
$$

will also image steep reflectors, because correlates the ascending wavefield of the source with the descending wavefield of the receptor.

Imaging conditions given by equations (50) and (51) will image reflectors reached by duplex waves, for example.

The next imaging condition, with only the ascending wavefields, is given by

$$
I_{U U}(y, x, z)=\sum_{\omega} S_{U}^{*}(\omega, y, x, z) \cdot G_{U}(\omega, y, x, z)
$$

and it can image reflectors which are affected by returning waves, for example.

Using all these imaging conditions, the final migration will be the sum of all images defined above. Thus, the final migrated imaged is obtained by:

$$
I=a_{1} I_{D D}+a_{2} I_{D U}+a_{3} I_{U D}+a_{4} I_{U U}
$$

where the values of the weight function $a_{j}$ (from $j=1,2,3,4$ ) have the purpose of weighting each image, as they have different amplitudes, resulting in a final image that may have a better balance in amplitudes and improvement in terms of imaging quality.

\section{NUMERICAL RESULTS}

Seismic modeling was tested using four velocity and seismic migration was demonstrated using two generated seismic datasets.

Initially, we tested the 2-D seismic modeling on a part of BP model, which has a salt body surrounded by low velocity strata, which is shown in Figure 2(a). An interesting comparison is presented between the results obtained with the seismic modeling method proposed in this work (Fig. 2(b)) and the results obtained by seismic modeling with an explicit finite difference method, which is shown in Figure 2(c). With the proposed technique, almost all descendents and ascendents events are taken into account. The only difference lies in the absence of modeled multiple reflections, since the second term of equations (23) and (24) was not considered. However, the modeling with full wave equation by finite difference method takes into account the effect of multiple scattering and all multiple reflection events. In Figures 2(b) and 2(c) the velocity model is overlaid to facilite the identification of the events (reflections, multiples, and others).

To demonstrate the proposed modeling procedure, three simple 3-D models were used to perform seismic modeling experiment. By convention, the depth direction $Z$ is positive down, $X$ is the direction "Inline" and $Y$ is the "Crossline" direction. Figure 3 shows the following models: (a) a plane, (b) the step (fault line), (c) a salt diapir and (d) the SEG-EAGE-3D model. The models (a), (b) and (c) have an associated velocity field with a minimum velocity of $2000 \mathrm{~m} / \mathrm{s}$ and a maximum velocity of $4000 \mathrm{~m} / \mathrm{s}$, with spatial grid defined by $n z=100$ points, $n x=n y=141$ points, spaced by $d x=d y=d z=10 \mathrm{~m}$. In the step model, the vertical reflector separate a strong lateral velocity variation. For the salt diapir the velocity model consists of a high velocity cylinder $(4000 \mathrm{~m} / \mathrm{s})$, surrounded by a low velocity equal to $2000 \mathrm{~m} / \mathrm{s}$. The cylinder body rests on a platform with a velocity of $4000 \mathrm{~m} / \mathrm{s}$. Both models were created with the purpose to generate duplex waves during the modeling process. The duplex waves generation phenomenon can be observed on these two models but it can be easily identified on the snapshots taken from the step model. These duplex waves, if they are correctly treated during the imaging procedure, will help to image the vertical reflectors better. The last model, the SEG-EAGE-3D model, has a complex structurally velocity model, as it is shown in Figure 3(d). It has a velocity grid with $n z=201$ points and $n x=n y=214$ points, spaced by $d z=20 \mathrm{~m}$ and $d x=d y=40 \mathrm{~m}$.

The modeling results from these described models were obtained with a shot injected approximately in the middle of the models. The time sections with receivers located over the entire surface to model, defined by $X, Y, z=0$, were obtained and 

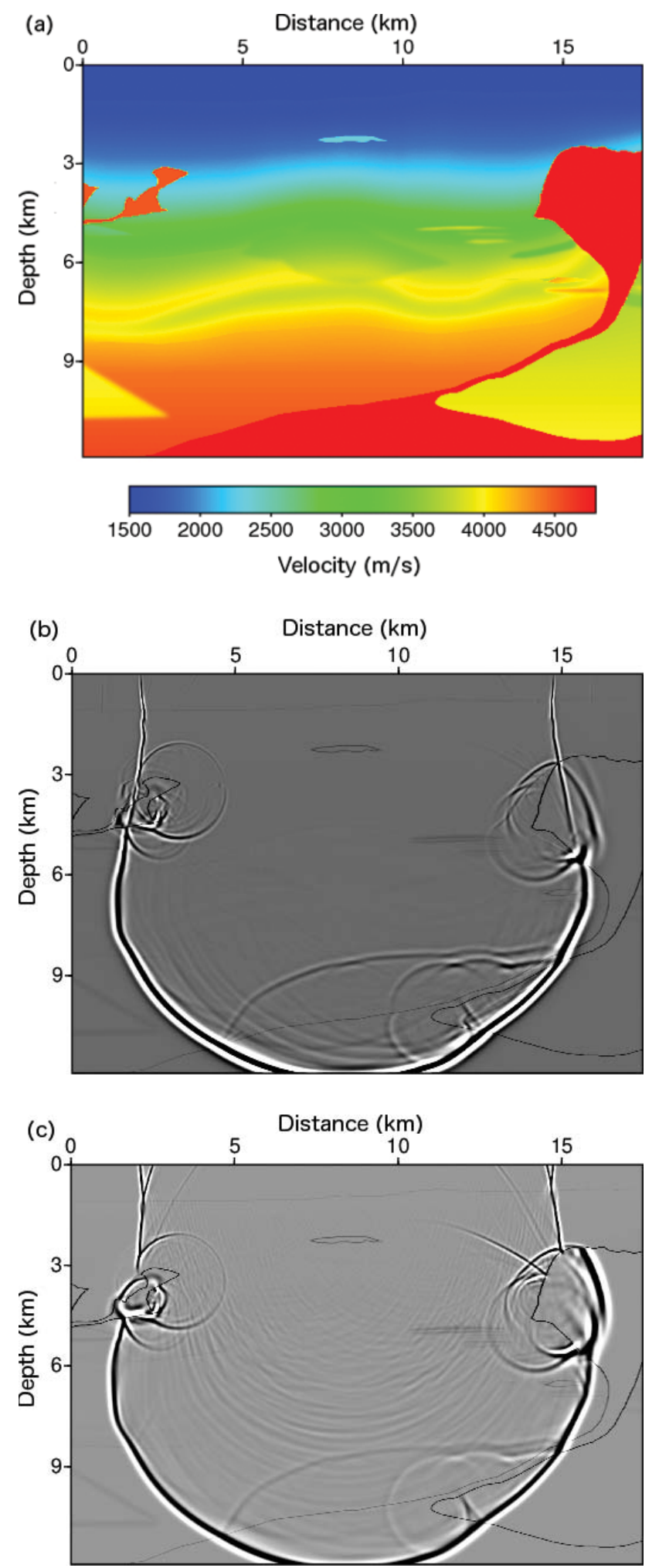

Figure 2 - Patch from 2-D BP velocity model (a); Snapshot of a modeled shot with the wave equation in its decoupled unidirectional components (b); Snapshot of a shot with the full wave equation solved by the finite difference method (c). 
(a)

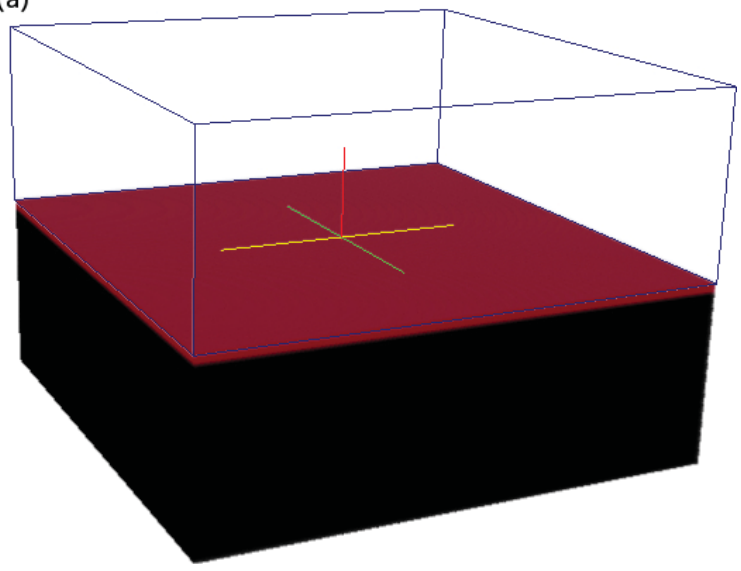

(c)

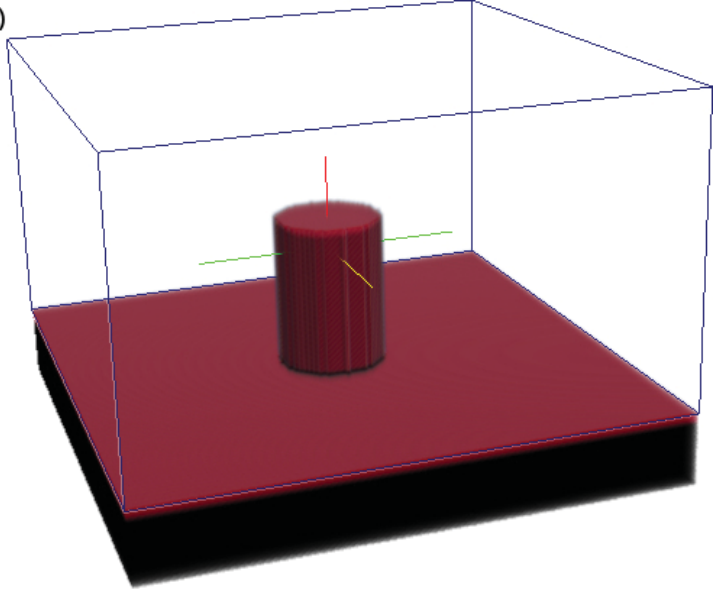

(b)
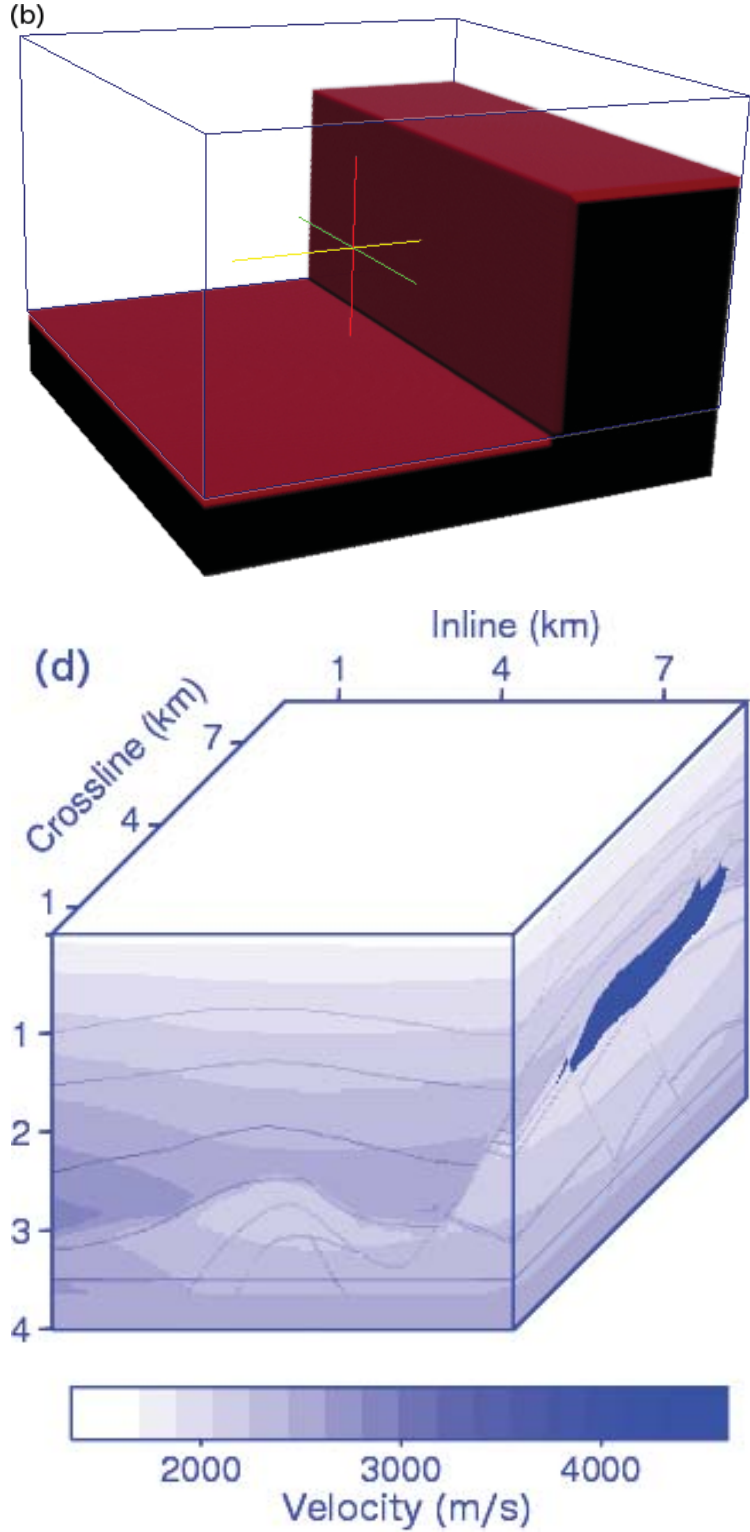

Figure 3 - 3-D velocity models: plane model (a); step model (b); the diapir model (c); and SEG-EAGE-3D velocity model (d).

snapshots during the propagation of the wavefield in these models, were saved to observe the seismic events.

Figure 4 shows the result of the 3-D seismic modeling on the plane reflector model. Figure 4(a) shows the wavefield at the instant of time $t=0.3 \mathrm{~s}$ and $t=0.6 \mathrm{~s}$. The figure in the top, was taken for a fixed depth along the $X-Y$ plane. The two figures below, were taken for fixed horizontal positions $X$ and $Y$. In these two last figures, we can observe wavefronts that propagate down and wavefront (reflected wave) that propagates upward. The wavefronts are marked in the figures and identified, for example: $D-0.3 s$ corresponds to a descending wave ( $D-$ Downward) at time equal to $0.3 s . R-U-0.6 s$ corresponds to a reflected and upward wavefront ( $U-$ Upward) at time $0.6 s$ and $D-0.6 s$ is a downward wavefront at time equal to $0.6 \mathrm{~s}$. We can observe also that $R-U-0.3 \mathrm{~s}$ is a ascending reflected wavefront in the time equal to $0.3 s$, and also that $T-D-0.3 s$ is a transmitted and descending wavefront at time equal to $0.3 \mathrm{~s}$. These figures clearly shows reflected and transmitted waves at different times during the propagation modeling procedure. The flat interface is marked at $0.5 \mathrm{~km}$ and it helps to visualize the right position of the wavefronts hitting the interface. Figure 4 (b) presents the seismograms. Two time sections are shown: in the below part, are 

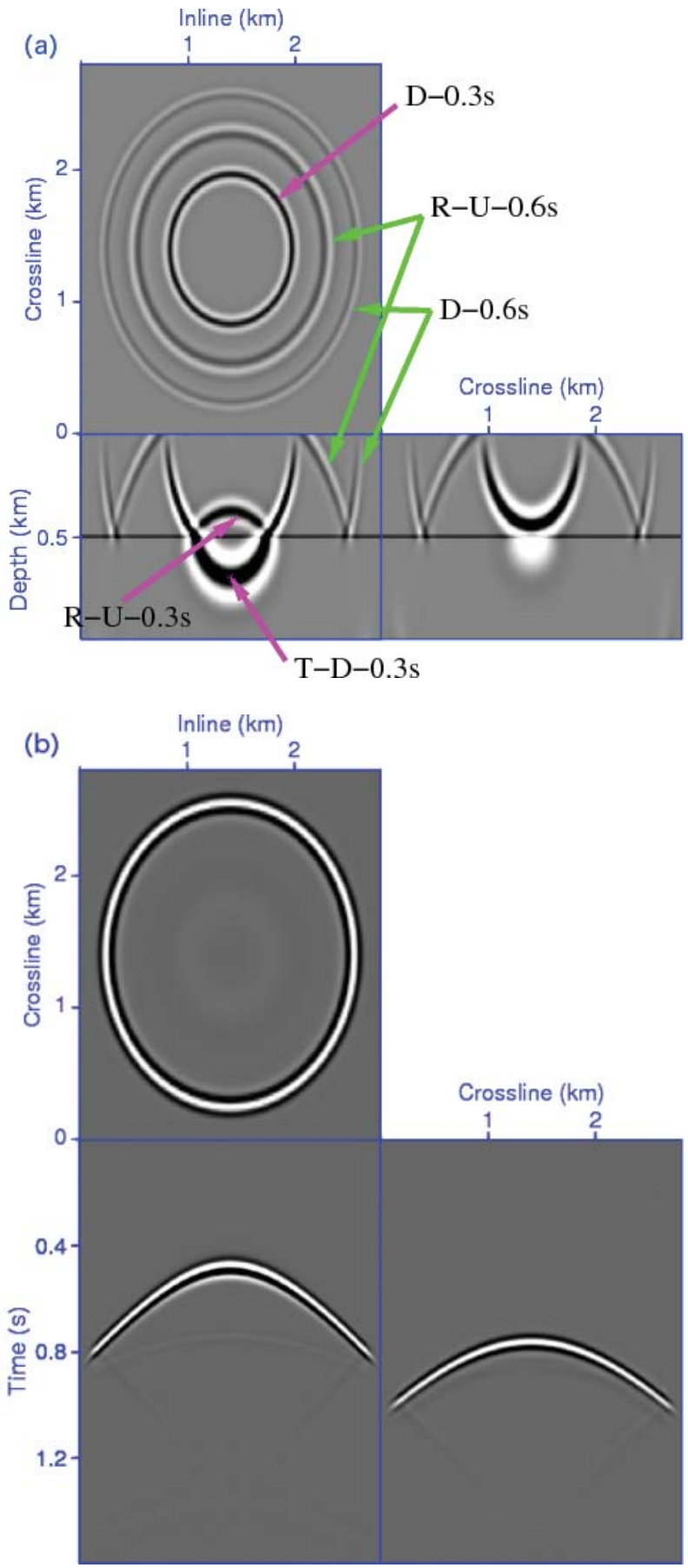

Figure 4 - Seismic modeling result of a shot using the 3-D SS-PSPI-UP-DOWN operator on the plane model. (a) Snapshots: fixed depth (top) and constant $X$ and $Y$ sections (bottom). (b) Snapshot at a fixed depth (top) and time sections taken along $X$ and $Y$ cross-section in the time section cube dataset.

the record of the ascending waves in the surface model for fixed horizontal directions $X$ and $Y$, in the top, a snapshot at $0.6 s$. As expected for a plane model, we note that the shape of the reflected wave in time is a hyperboloid of revolution with its apex at the position of the shot. Meanwhile, the snapshot for a fixed depth cut, shows a wavefront with a shape of a perfect circle because the wave was propagating in a medium that does not have velocity variation along the horizontal directions. 

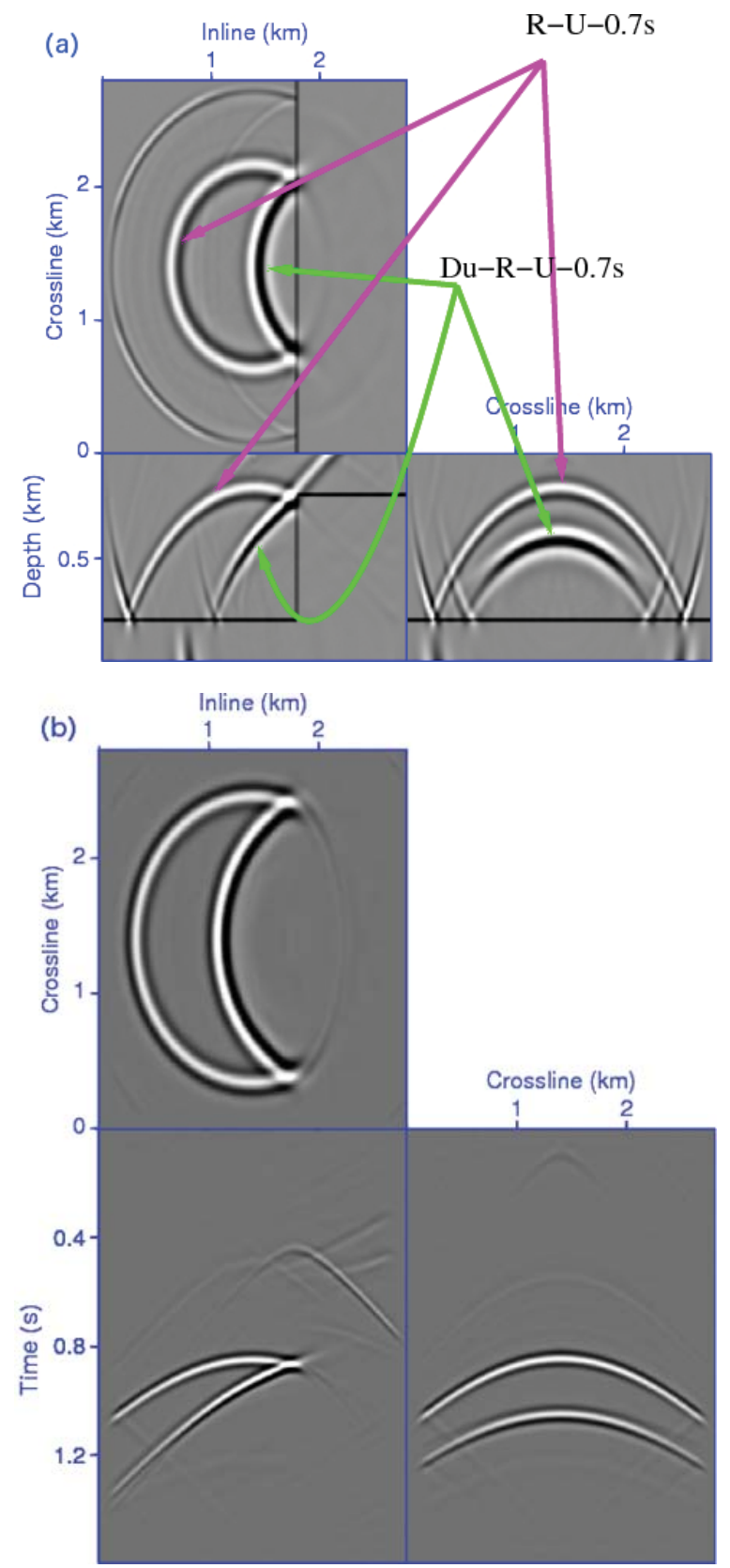

Figure 5 - Seismic modeling result of a shot using the 3-D SS-PSPI-UP-DOWN operator on the step model. (a) Snapshots: fixed depth (top) and constant $X$ and $Y$ sections (bottom). (b) Snapshot at a fixed depth (top) and time sections taken along $X$ and $Y$ cross-sections in the time section cube dataset.

The result of 3-D seismic modeling on the step model (Fig. 3(b)), clearly shows duplex waves, Figure 5(a). These waves are generated by the reflection in the bottom horizontal reflector followed by a reflection in the vertical reflector. In this model, the vertical reflector is inside a medium with a strong lateral variation velocity to enhance the generation of these waves. Also, the following events are flagged propagated upwards: $R-U-0.7 \mathrm{~s}$ is the reflected wave at $0.7 s$ and $D u-R-U-0.7 s$ is a duplex wave $0.7 s$ after the injected the shot. Already in Figure 5(b) we can sees three slices that are the data recorded on the surface of the model. The arrival of the duplex wave right after the registration of the reflected wave is clearly noted.

The result of 3-D seismic modeling on the diapir salt model is shown in Figure 6. Figure 6(a) shows three cuts and one ex- 

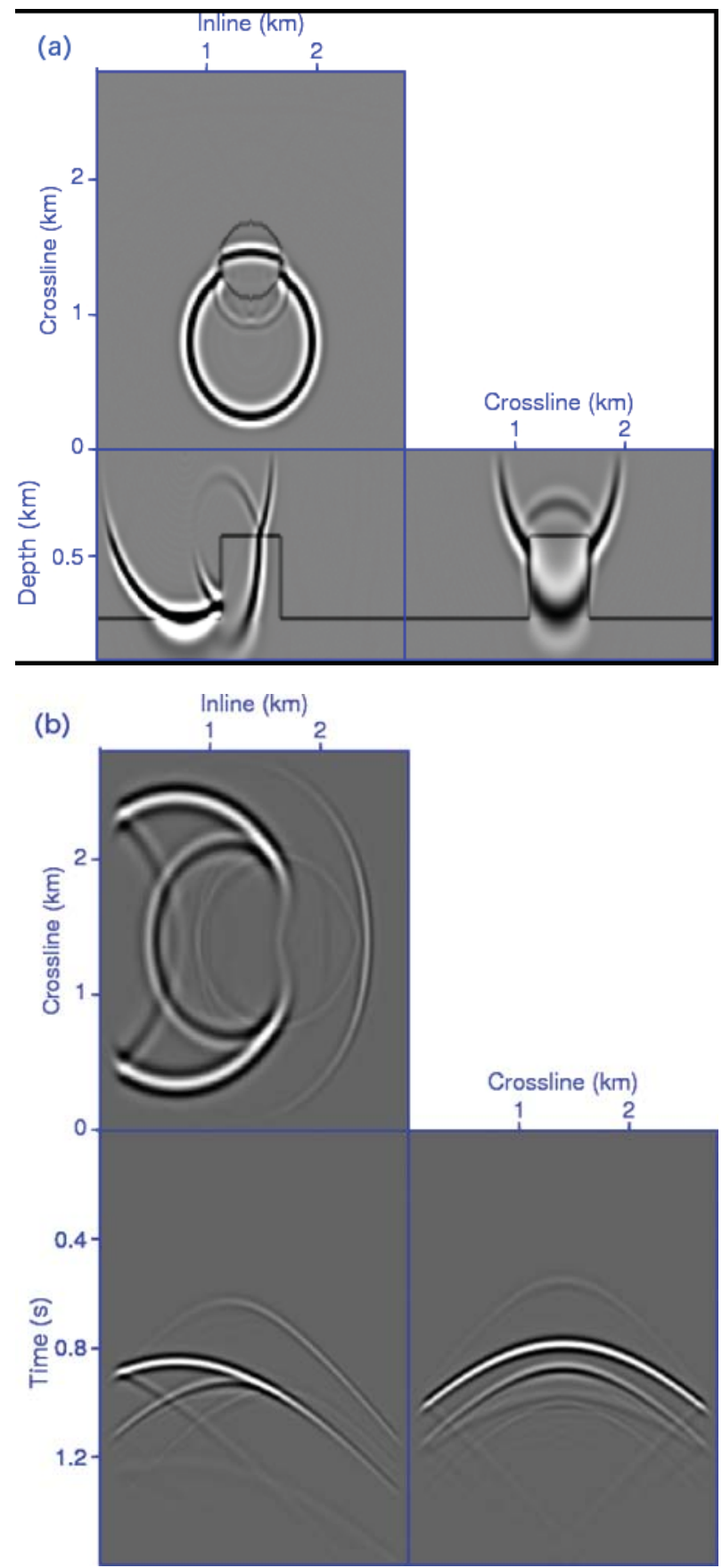

Figure 6 - Seismic modeling result of a shot using the 3-D SS-PSPI-UP-DOWN operator on the diapir salt model. (a) Snapshot at 0.3 (top) for a fixed depth and constant $X$ and $Y$ sections (bottom). (b) Snapshot at a fixed depth (top) and time sections taken along $X$ and $Y$ cross-sections in the time.

tra snapshot at $t=0.3 \mathrm{~s}$. In depth and cross cutting sections, we can observe the propagation of wavefronts more quickly into the cylinder. in the data registered at the surface model, shown in Figure $6(b)$ and in the cross-sections of the seismogram, there is the presence of the reflected wave and the duplex wave, and also a reflection on the top of the cylinder. In the upper part of the figure a snapshot is shown. To get a better view of the wavefronts propagation, a sequence of snapshots in horizontal cut into uniform time intervals were saved, as shown in Figure 7. These snapshots show the propagation of the wavefront and the effects due to the 


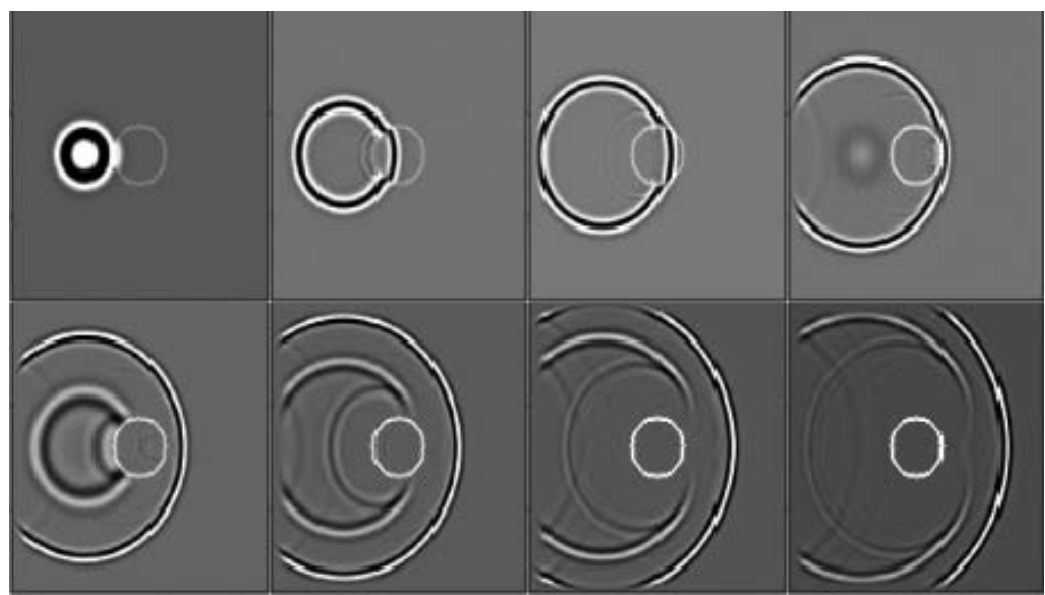

Figure 7 - Sequence of snapshots taken for a horizontal cut (fixed depth) in the 3-D diapir salt model.

high velocity contrast as well as the presence of weak edge effects because a damping factor along the border of the model, as proposed by Cerjan (Cerjan et al., 1985), was used. In the second row of snaps, we can identify the duplex wave and observe its propagation inside the velocity model.

To observe the propagation of the wavefronts in a complex medium with the presence of a salt body, we used the SEG-EAGE3D model and Figure 8(a) shows slices of three snapshots superimposed on reflectivity. The propagation in the salt body is clearly observed, where the wavefield travels with greater speed and interacts with complex structures outside the salt body. Around the body salt, complex propagations are observed and become more evident in the seismogram cuts and snapshot shown in Figure 8(b).

No effects of azimuthal anisotropy is observed in the 3-D seismic modeling tests using the unidirectional operator SS-PSPIUP-DOWN. Also seismograms show clear imaging with a low noise presence, making this type of modeling using coupled downgoing and upgoing waves very useful and eficient for modeling 3-D seismic models.

To test the 3-D pre-stack depth seismic migration proposed here 500 families of common shots were modeled using the step and diapir salt models. These shots were distributed evenly over the surface $X-Y$ at level $z=0$. Figure 9(a) shows the result of the pre-stack depth migration using the conventional 3-D SS-PSPI operator. In this case, only the descending source and receivers wavefields were taken into account and the imaging condition is given by equation (49). Cuts of the migrated cube toward "Inline", "Crossline" directions and in depth, show the horizontal reflectors imaged in their correct spatial location. However, the absence of vertical reflecting wall is evident in this result, therefore only the down propagated wavefields were taken into account and not those up propagated. Using 3-D PSPI-SS-UP-DOWN operator for pre-stack depth migration and the sum of the images from the conditions given by the equations (50), (51) and (52), the migrated image result is shown in Figure $9(b)$, where the vertical reflector is now rebuilt. The difference between these two results is very obvious, since the conventional migration method does not take into account the duplex wave. Some spurious events can be observed at the top due to the low density of the modeled shots.

The results of the depth migration of the dataset modeled on the diapir salt model is shown in Figures 10(a) and 10(b), with the 3-D SS-PSPI operator and the 3-D SS-PSPI-UP-DOWN operators, respectively. Although the propagation in this medium is more complicated due to the circular geometry of the diapir, the result of the migration with the 3-D SS-PSPI-UP-DOWN operators, show a correct reconstruction of the steep reflector, with $90^{\circ}$, and outlining the cylindrical boundary of the diapir. Moreover, conventional migration method result on these dataset, was not able to image the vertical reflector, because the standard operator only considers the propagation of the downgoing waves.

\section{CONCLUSIONS}

In this paper, we present a 3-D technique for seismic modeling and pre-stack depth migration based on the unidirectional wave equation solution.

The propagation of the wavefield is performed using the two unidirectional equations, obtained from the decoupling of the full acoustic wave equation. These unidirectional equations take into account the propagation of the ascending and descending wavefield separately. However, multiple scattering events are not taken into consideration during the extrapolation procedure. Huygens's 

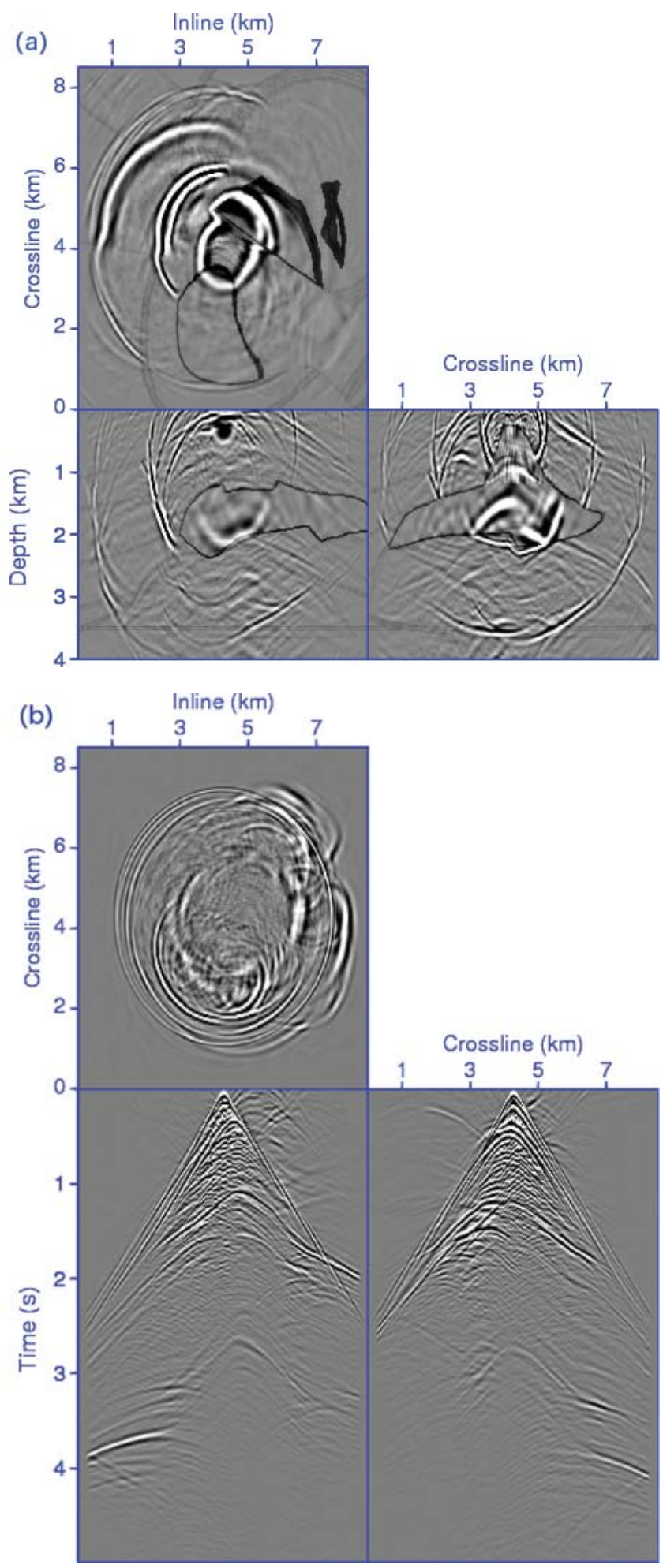

Figure 8 - Seismic modeling result of a shot using the 3-D SS-PSPI-UP-DOWN operator on the SEG-EAGE3D model. (a) Snapshots: fixed depth (top) and constant $X$ and $Y$ sections (bottom). (b) Snapshot at a fixed depth (top) and time sections taken along $X$ and $Y$ cross-sections in the time section cube dataset. 

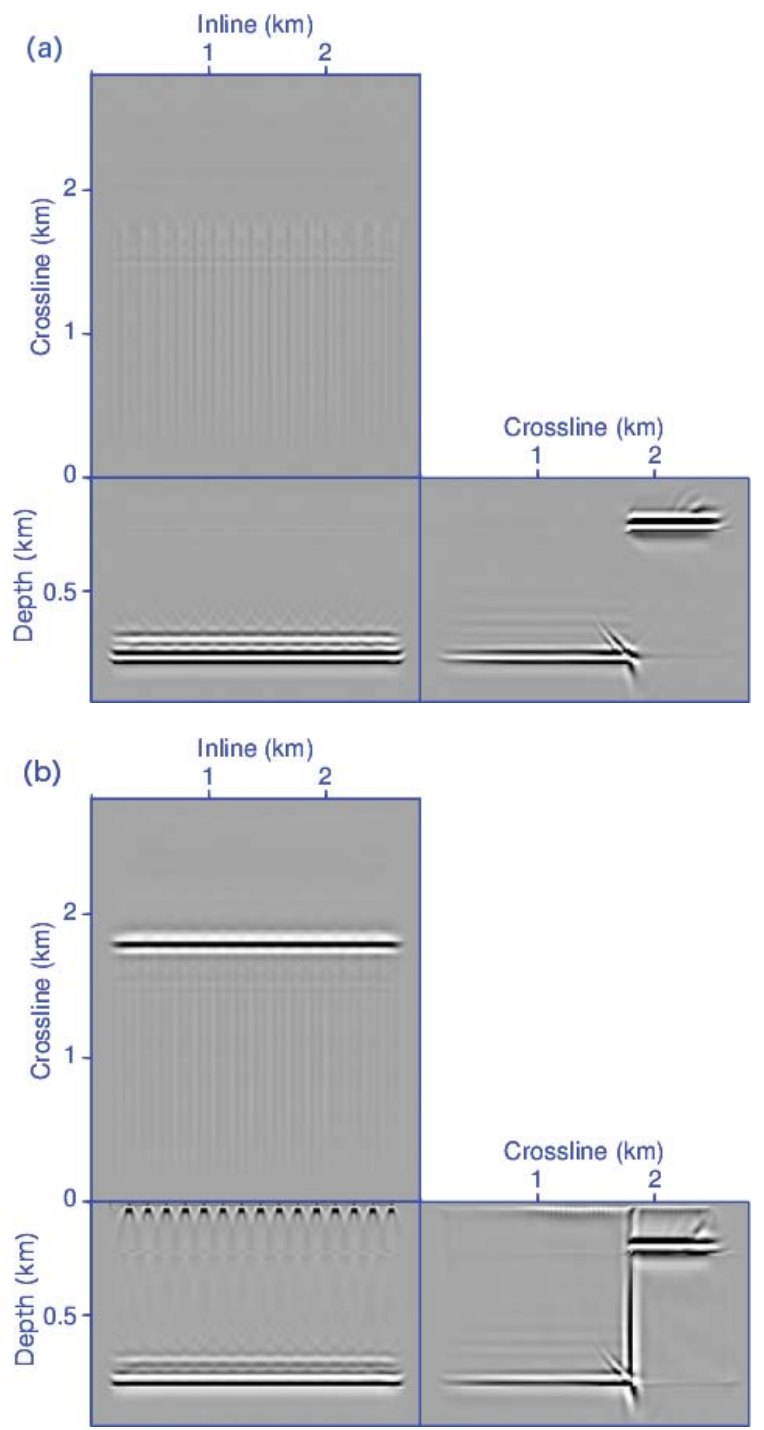

Figure 9 - Pre-stack depth migration of the step model with the 3-D PSPI-SS operator (a) and with the 3-D PSPI-SS-UP-DOWN operator (b).

principle is appropriately applied during the propagation of both upwards and downwards wavefields.

Due to its robustness and computational efficiency, the 3D unidirectional SS-PSPI operator is used to make the upward and downward extrapolation of the source wavefield during the modeling procedure for source and receivers for migration. This 3-D operator named SS-PSPI-UP-DOWN has been proven to be suitable for propagation in complex 3-D media and without azimuthal anisotropy effects.

The sections modeled with the 3-D SS-PSPI-UP-DOWN operator show seismograms with good quality and without the presence of numerical dispersion, including models of complex structure as the SEG-EAGE-3D. The snapshots show the correct
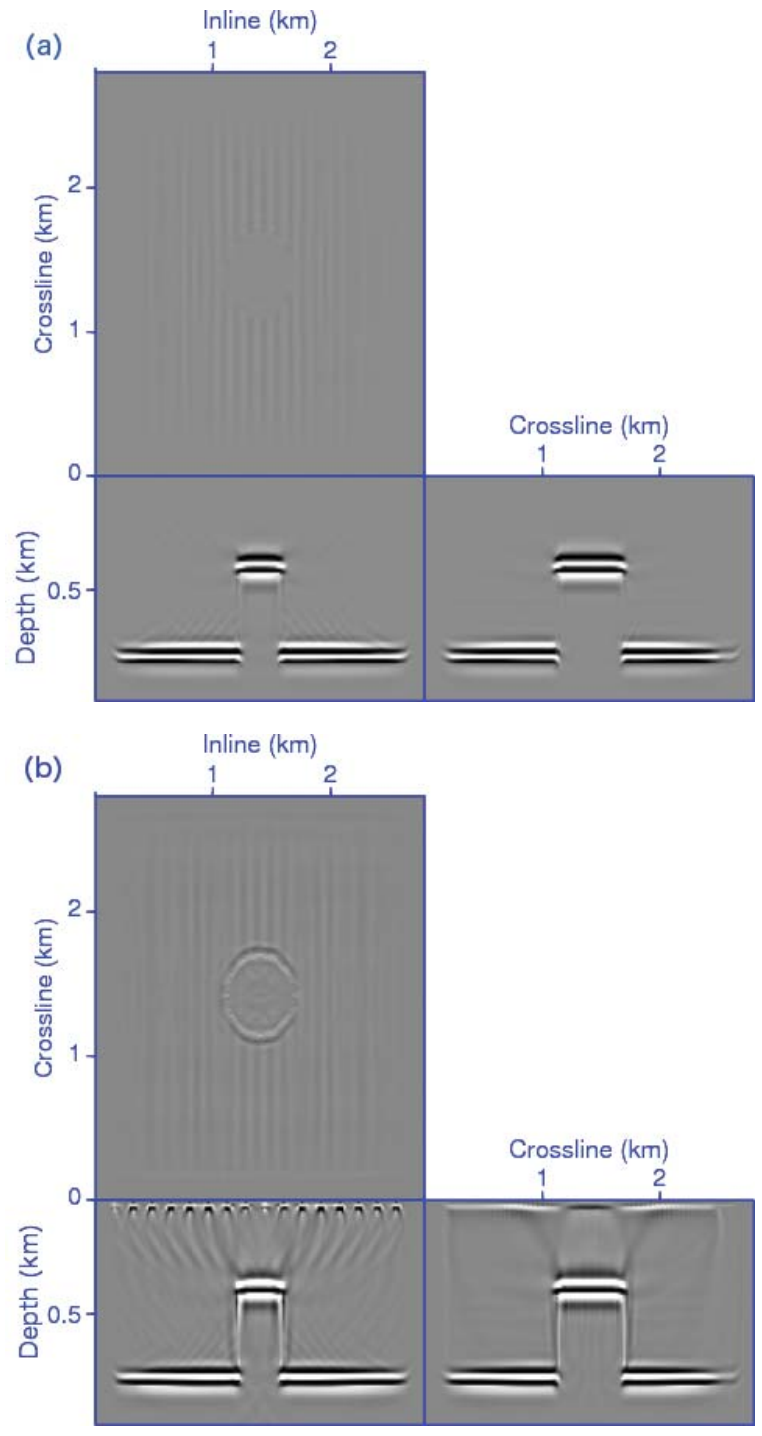

Figure 10 - Pre-stack depth migration of of diapir salt model with the 3-D SSPSPI operator (a) and with the 3-D data SS-PSPI-UP-DOWN operator (b).

propagation of the wavefronts, both up and down, thereby simulating one full wave propagation.

3-D seismic migration was performed to validate the seismic modeling algorithm and two datasets of pre-stacked were used. The results of the migration with the 3-D SS-PSPI-UP-DOWN operator show reflectors well imaged, including those associated with strong lateral velocity contrast and also good imaging of vertical reflectors due to the correct treatment of "duplex" waves during the extrapolation procedure. Thus, 3-D SS-PSPI-UPDOWN operator was demonstrated to be superior to the conventional 3-D SS-PSPI operator and constitutes an efficient alternative to image steep reflectors, having a great potential to be applied in real 3-D data. 


\section{REFERENCES}

ALDUNATE GC \& PESTANA RC. 2006. Método Híbrido de Migração Pré-Empilhamento em Profundidade no Domínio da Freqüência em duas Etapas com Interpolação. Brazilian Journal of Geophysics, 24(1): 91-102.

ALDUNATE GC \& PESTANA RC. 2010. Migração 3-D em profundidade usando o operador Split-Step Phase-Shift Plus Interpolation: Uma comparação de dois métodos estatísticos para a seleção das velocidades de referência. In: Simpósio Brasileiro de Geofísica, 4., Proceedings... Brasília, DF, Brazil: SBGf. CD-ROM.

ALDUNATE GC \& PESTANA RC. 2011. Migração 3-D pé-empilhamento em profundidade com os métodos SS-PSPI e RTM: um experimento usando os dados 3-D SEG-EAGE. In: International Congress of the Brazilian Geophysical Society, 12., Rio de Janeiro, Brazil: SBGf, CDROM.

AMUNDSEN L, ARNTSEN B, REITAN A, DISCHLER E \& URSIN B. 2008. Wave equation depth migration - a new method of solution. SEG Annual Meeting, Expanded Abstracts, Las Vegas, USA, p. 2252-2256.

CARCIONE JM, HERMAN GC \& TEN KROODE APE. 2002. Seismic modeling. Geophysics, 67: 1304-1325.

CERJAN C, KOSLOFFD, KOSLOFF R \& RESEF M. 1985. A non-reflecting boundary condition for discrete acoustic and elastic wave equations. Geophysics, 50: 705-708.

CHEN JB \& DU SY. 2010. Kinematic characteristics and the influence of reference velocities of phase-shift-plus-interpolation and extended-
split-step-Fourier migration methods. Geophysical Prospecting, 58: 429-439.

CLAERBOUT JF. 1985. Imaging the Earth's interior. Blackwell Scientific. $300 \mathrm{pp}$.

JIN S \& XU S. 2006. One-return wave equation migration. Imaging of duplex waves. Presented at the SEG 76th Annual Meeting.

THORBECKE J. 2012. 2D finite-difference wavefilled modelling. Available on: <http://janth.home.xs4all.nl/Software/fdelmodcManual.pdf >. Access on: April ?, 2013.

WU Y, HE Z \& HUANG D. 2007. Wave-field modeling of common shot gather records based on Huygens's principle. Available on: $<$ http://www.paper.edu.cn>. Access on: April ?, 2013.

XIAOJUN X, ZHENHUA H \& DEJI H. 2006. One-way wave equation seismic prestack forward modeling with irregular surfaces. Applied Geophysics, 3: 13-17.

YE RC \& JIA XF. 2012. An effective denoising strategy for wave equation migration based on propagation angles. Applied Geophysics, 9: 33-40.

ZHANG W \& WONG YS. 2010. Efficient parallel hybrid computations for threedimensional wave equation prestack depth imaging. International Journal of Numerical Analysis and Modeling, 7: 373-391.

ZHANG Y, XU S \& ZHANG G. 2006. Imaging complex salt bodies with turning-wave one-way wave equation. SEG Annual Meeting, Expanded Abstracts, New Orleans, Louisiana, USA, p. 2323-2327.

\section{NOTES ABOUT THE AUTHORS}

Gary Corey Aldunate holds a B.Sc. degree in Physics from Universidad Autónoma Tomás Frías (1997) and M.Sc. degree in Applied Geophysics from Universidade Federal da Bahia (2002). Specialist on seismic wave propagation, including seismic migration in heterogeneous media, is a member of Brazilian Geophysical Society (SBGf) and Society of Exploration Geophysicists (SEG).

Reynam da Cruz Pestana is an associate professor at the Department of Physics of the Earth and Environment of the Physics Institute of Universidade Federal da Bahia (UFBA) since 1991. Received B.Sc. in Physics in 1984 and Ph.D. in Geophysics in 1988, both degrees from UFBA. Has a post-doc at Karlsruhe University from 1988 to 1991 and at the University of Texas at Austin from 1998 to 1999. Expertise in Applied Geophysics, working mainly in processing and imaging of seismic data, developing seismic processing methods for complex geologic problems. Recently, has developed RTM algorithms for VTI and TTI media using pseudo acoustic methods. Is also a recipient of the Brazilian Geophysical Society's Nero Passos award: Educational and Research Geophysicist. Is a member of Brazilian Geophysical Society (SBGf), Society of Exploration Geophysicists (SEG) and the European Association of Geoscientists and Engineers (EAGE). 